\title{
Cytology of Colonic Mucosal Secretions from Patients with Non-specific Haemorrhagic Proctocolitis in Complete Clinical Remission
}

\author{
POVL RIIS,* M.D.; FLEMMING VALDORF-HANSEN, $\dagger$ M.D.; POUL ANTHONISEN, $\dagger$ M.D.
}

Brit. med. F., 1966, 1, 712-714

As a rule the diagnosis of non-specific haemorrhagic proctocolitis is easy when the case history is characteristic and when the patients can be examined during an active phase of the disease. In the majority of such cases sigmoidoscopy will reveal typical changes, as will radiological examination if a substantial part of the colon is involved in the pathological process. The diagnosis can, however, present considerable difficulty if the colon symptoms are slight and uncharacteristic-for example, comprising only rectal bleeding in connexion with constipation. Furthermore, the patients may be quite free of symptoms at the time of examination.

In this situation it is desirable to reduce the diagnostic threshold by the introduction of microscopical criteria. Of the different techniques devised for this purpose the suctionbiopsy method of Truelove, Horler, and Richards (1955) has been the most popular. We have described a simple replica method (Anthonisen and Riis, 1961) which has proved a valuable tool in the diagnosis of proctosigmoidal disease.

In preparations from patients with active non-specific haemorrhagic proctocolitis unequivocal signs of inflammation have always been found. In contrast, preparations from healthy subjects and from patients with the irritable colon syndrome have usually contained no inflammatory cells at all. Occasionally a few granulocytes have been present singly or in isolated small clusters, but the quantitative distinction from the findings in proctocolitis has always been clear (Anthonisen and Riis, 1962 ; Anthonisen, Hvidberg, and Riis, 1963).

The present work intends to compare the diagnostic thresholds of the replica method and macroscopic sigmoidoscopy respectively in a group of patients with non-specific haemorrhagic proctocolitis in complete clinical remission.

\section{Material and Methods}

The material comprises 65 episodes in 46 patients from the out-patient proctocolitis clinic. Thirty-two patients were women (aged 14 to 74 , average 43 years) and 14 were men (aged 20 to 71 , average 36 years). In 23 patients the disease was confined to the rectum, in six the inflammation extended into the sigmoid flexure, and in 17 a greater part of the colon was involved, the extension of the disease having been elicited by sigmoidoscopy and radiological examination of the colon with Welin's double-contrast technique (Kinsey, Hornnes, Anthonisen, and Riis, 1964). No selection was carried out, the cases simply representing a consecutive series of patients reporting for control during complete clinical remission of the disease -that is, patients with two or less normal stools daily, with no bleeding or purulent discharge from the rectum, and with no abdominal discomfort.

None of the patients included received any treatment at the time of the episodes concerned.

Sigmoidoscopy was performed by one of three experienced examiners. In the macroscopical evaluation importance was attached to the absence or presence of granulation, vulnerability (friability), haemorrhage, mucopus in the lumen, erosions,

* Medical Department B, Gentofte Hospital, Denmark. t Medical Department B, Glostrup Hospital, Denmark. ulcerations, pseudopolyps, abnormal vascular patterns, and scarring.

Mucosal replicas were obtained, the technique previously described (Anthonisen and Riis, 1961) being used. As a rule two specimens were taken from separate sites at different levels of the rectum, always including possible areas of macroscopically abnormal mucosa. In the microscopical evaluation total cellularity and the relative representation of erythrocytes, lymphocytes, and the different types of granulocytes were noted. In case of discrepancy between two corresponding specimens the one with most pronounced inflammatory changes was chosen to represent the episode.

As a rule the microscopical evaluation was made "blindly." The description was graduated according to the following criteria :

(1) Total cellularity

$0=$ no leucocytes present.

$(t)=$ less than 20 leucocytes present.

+ and $++=$ intermediate stages.

$+++=$ leucocytes seen throughout the preparation.

(2) Admixture of erythrocytes is expressed by 0 to +++ according to the same criteria as in (1).

(3) Relative representation of neutrophils and lymphocytes ranging from 0 (absent) to +++ (dominating cell type).

(4) Occurrence of eosinophils (and occasional basophils) is expressed by:

$$
\begin{aligned}
0= & \text { no eosinophils present } ; \\
+= & \text { eosinophils present, but only to the extent seen in } \\
& \text { colonic inflammations other than non-specific haemor- } \\
& \text { rhagic proctocolitis (diverticulitis, salmonella- } \\
& \text { enterocolitis, toxic colitis); } \\
++= & \text { intermediate stage } ; \\
+++= & \text { eosinophils constitute about one-fourth or more of all } \\
& \text { leucocytes. }
\end{aligned}
$$

\section{Overall Characteristics}

Concerning disease activity the principle of graduation is shown in Table I. Occurrence of erythrocytes alone has not been taken as a sign of inflammatory activity, because handling of the sigmoidoscope can provoke bleeding even in a non-inflamed mucosa. With regard to local inflammatory eosinophilia, this is noted if a relative number of eosinophils of more than grade + is demonstrated in at least one preparation.

TABLE I.-Principle for Graduation of Disease Activity on the Basis of

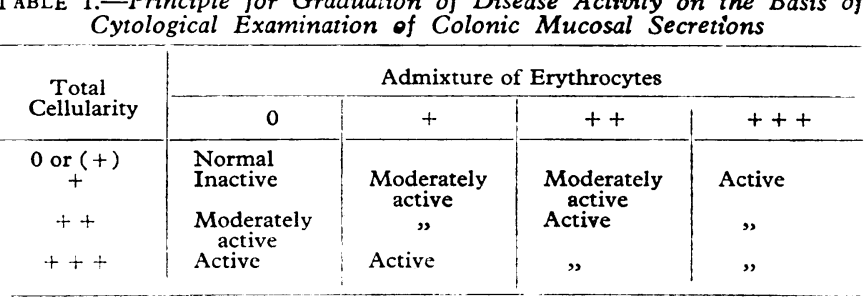

Results

The results are shown in Table II. The abnormal sigmoidoscopical findings comprised granularity (23 episodes), vulner- 
ability (friability) (11 episodes), ulcerations (2 episodes), scarring (2 episodes), and submucosal haemorrhages (2 episodes). In other words, the macroscopical evidence of active disease was slight in most cases.

Table III shows the relation between cytological pictures and lengths of clinical remissions.

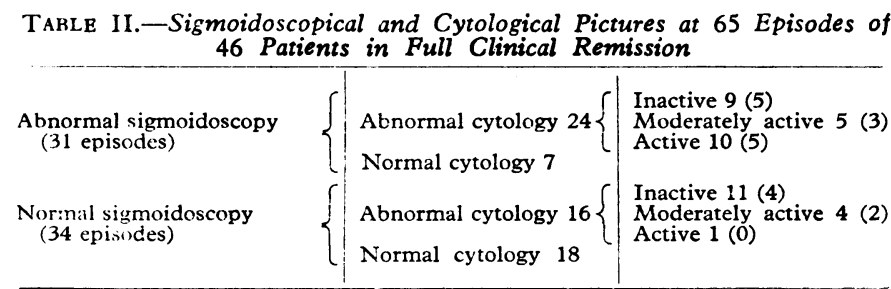

Figures in parentheses indicate number of cases with local inflammatory eosinophilia.

TABt. III.-Relation between Cytological Pictures and Lengths of Clinical Remissions

\begin{tabular}{|c|c|c|}
\hline \multirow{2}{*}{ Length of Remission } & \multicolumn{2}{|c|}{ Cytolegical Picture } \\
\hline & Abnormal & Normal \\
\hline $\begin{array}{l}\text { Less than } 6 \text { months ( } 49 \text { episodes) } \\
\text { More than } 6 \text { months ( } 16 \text { episodes) }\end{array}$ & $\begin{array}{c}32(14) \\
7 \text { (3) }\end{array}$ & $\begin{array}{c}17(12) \\
9(7)\end{array}$ \\
\hline
\end{tabular}

Figures in parentheses indicate number of episodes with a normal sigmoidoscopical finding.

\section{Discussion}

In the present investigation, which comprised patients with verified proctocoltis in full clinical remission, unequivocal microscopical signs of mucosal inflammation were demonstrated in about $50 \%$ of the cases whose mucosa, at sigmoidoscopy, appeared to be normal macroscopically.

lior the patients in this series in whom the diagnosis had been made in an earlier and more active phase of the disease that observation was, of course, of no importance for the differential diagnosis. The finding suggests, however, that microscopical examination (cytological preparation, biopsy) can be of definite diagnostic value in patients who are seen for the first time in a symptom-free or symptom-poor phase of the disease and who present a debatable or normal sigmoidoscopical picture.

The ability of microscopical methods to diagnose colonic mucosal inflammation in patients with slight or no intestinal symptoms will also make such methods valuable when the true incidence of proctocolitis in the population at large or in selected groups-as, for example, in patients with ankylosing spondylitis or erythema nodosum-is sought.

Our finding that definite microscopical signs of mucosal inflammation could be demonstrated in some patients several months after subsidence of symptoms, even when the mucosa appeared macroscopically normal at sigmoidoscopy, underlines the necessity for also using microscopical methods when describing the natural history of non-specific haemorrhagic proctocolitis.

It is still too early to evaluate the prognostic significance of the combination, normal sigmoidoscopy/abnormal cytology versus normal sigmoidoscopy/normal cytology, in clinically remitted cases, as the number of relapses a year is often very low.

In the present investigation no evaluation of the degree of remission obtained by treatment was intended. As a rule no objective method is necessary for this purpose, as the aim of a symptomatic treatment (which is the only type of treatment as yet known) will normally be a clinical remission irrespective of possible residual inflammatory activity. Microscopical methods may, however, turn out to be useful in determining the fitness of the rectal stump for secondary ileorectal anastomosis after subtotal colectomy.
As can be seen in Table II, in about half of our cases the cytological method revealed only inflammation as such. In the remaining cases, however, definite inflammatory eosinophilia strongly suggested the diagnosis of non-specific haemorrhagic proctocolitis, which is the only colon disease-at least in Denmark-in which local eosinophilia has been found characteristic (Anthonisen and Riis, 1962 ; Riis and Anthonisen, 1964).

Of course segmental, especially right-sided, proctocolitis escapes diagnostic evaluation by the present methods of investigation.

Several other investigators have compared the clinical condition of proctocolitis patients with the macroscopical appearance of the colonic mucosa at sigmoidoscopy and the inflammatory activity as judged by microscopical examination with the use of mucosal biopsy technique.

Lumb and Protheroe (1955), in a series of ulcerative colitis cases of all degrees of clinical activity, found 11 in which characteristic histological changes were present in spite of the normal appearance of the mucosa at sigmoidoscopy.

Truelove and Richards (1956) investigated 42 patients. In 16 fully remitted cases the sigmoidoscopical picture was normal, and in six of these abnormal histology was demonstrated.

In a follow-up study Dick and Grayson (1961) compared sigmoidoscopical findings, mucosal biopsies, and clinical conditions of 62 proctocolitis patients. Among 43 of these who had been free of symptoms for from a few months to more than two years 19 showed a macroscopically normal mucosa at sigmoidoscopy. Biopsies from 10 were normal, nine were abnormal. When the remission had lasted less than six months there was almost complete accordance between sigmoidoscopical picture and biopsy, whereas approximately $60 \%$ of patients having longer remissions showed signs of inflammatory activity at biopsy, in contrast to a little more than $40 \%$ with macroscopically abnormal mucosa. In the present series signs of inflammatory activity were demonstrated in the cytological preparations in at least two-fifths of the episodes with remissions for more than six months.

In a larger series of proctocolitis patients Matts (1961) studied 47 "quiescent" cases of which about $50 \%$ showed a macroscopically abnormal mucosa and approximately $80 \%$ a pathological histological picture. In $15 \%$ both sigmoidoscopy and biopsy gave normal results. It seems that not all of Matts's "quiescent" cases were in complete clinical remission.

Flick, Voegtlin, and Rubin (1962), on the basis of 144 biopsies from 47 patients with ulcerative colitis, concluded that they had never seen a clearly abnormal biopsy from a patient who presented a macroscopically normal mucosa at sigmoidoscopy.

The diagnostic value of microscopical methods in clinically remitted cases of non-specific haemorrhagic proctocolitis is thus disputed. In the present investigation the sampling method revealed definite signs of mucosal inflammation in nearly half the cases in which no macroscopical abnormality was seen at sigmoidoscopy.

The paper of Baron, Connell, and Lennard-Jones (1964) dealing with the difficulties in describing mucosal appearances in proctocolitis patients has an important bearing on the finding in our investigation of normal cytological pictures in seven cases which presented a macroscopically abnormal mucosa at sigmoidoscopy. The explanation of this fact may be that slight macroscopical abnormalities-as, for example, granularity-are diagnosed with uncertainty. It seems, however, that some relation exists between cytological and macroscopical signs of inflammation. In the cases with abnormal sigmoidoscopical pictures the majority of the cytological preparations showed moderately active to active inflammation, whereas a normal sigmoidoscopical finding most often corresponded with a cytological preparation showing inflammation of very low activity. 
The main reason for discrepancy between the two methods of evaluation thus seems to be a difference of threshold.

\section{Summary}

Sixty-five episodes of complete clinical remission in 46 patients with a verified diagnosis of non-specific haemorrhagic proctocolitis were studied with respect to macroscopic and microscopical signs of mucosal inflammation.

The microscopical evaluation was based on the cytological method described in a previous paper. Macroscopic assessment was made by sigmoidoscopy with the use of conventional criteria.

In 31 episodes sigmoidoscopy revealed macroscopically abnormal mucosas; in 24 of these the cytological preparations also showed inflammatory changes, while the cytological pictures were normal in seven. In the remaining 34 episodes no mucosal changes were found at sigmoidoscopy ; in 16 of these distinct inflammation was seen microscopically, while normal cytology was demonstrated in 18 .

In about two-thirds of the episodes of patients with remissions shorter than six months the cytological method showed inflammatory changes, whereas the corresponding proportion in remissions of more than six months' duration was only slightly over two-fifths.
The ability of microscopical techniques-like the cytological method used in the present investigation-to demonstrate unequivocal signs of inflammation in proctocolitis patients who are in full clinical remission, and who may even show a normal sigmoidoscopical picture, can be utilized diagnostically when such patients are first seen in a symptom-free or symptom-poor phase. The fact also points to the latent chronicity of nonspecific haemorrhagic proctocolitis and calls for circumspection with regard to the prognosis of the disease.

\section{REFERENCE}

Anthonisen, P., Hvidberg, E., and Riis, P. (1963). Ugeskr. Laeg., 125, 501.

— and Riis, P. (1961). Lancet, 2, 81.

- (1962). Acta med. scand., 172, 375.

Baron, J. H., Connell, A. M., and Lennard-Jones, J. E. (1964). Brit. med. 7., $1,89$.

Dick, A. P., and Grayson. M. J. (1961). Ibid., 1, 160.

Flick, A. L., Voegtlin, K. F., and Rubin, C. E. (1962). Gastroenterology, 42, 69i.

Kinsey, I., Hornnes, N., Anthonisen, P., and Riis, P. (1964). Acta med. scand., 176, 181.

Lumb, G., and Protheroe, R. H. B. (1955). Lancet, 2, 1208.

Matts, S. G. F. (1961). Quart F. Med., 30, 393.

Riis, P., and Anthonisen, P. (1964). Acta med. scand., 175, 85.

Truelove, S. C., Horler, A. R., and Richards, W. C. D. (1955). Brit. med. F., 2, 1590 .

- and Richards, W. C. D. (1956). Ibid., 1, 1315.

\title{
Functional and Structural Studies of Small Bowel in Ankylostomiasis
}

\author{
B. N. TANDON,* M.D. ; B. C. DAS $\dagger$ M.B., B.S. ; A. K. SARAYA, $\ddagger$ M.D. ; M. G. DEO,§ M.D.
}

Brit. med. F., 1966, 1, 714-716

Ankylostomiasis is a widely prevalent problem of tropical countries. Several aspects of this malady remain to be investigated (W.H.O., 1959), and one of these pertains to the functional and structural changes of the small bowel. In the early part of this century Ashford and King (1907) and Whipple (1909) independently reported intestinal damage in necropsy studies of hookworm-infested subjects. This was later supported by Rotter (1931), who, from post-mortem studies, gave a detailed description of histological changes of the small gut in ankylostomiasis. In recent years the availability of a simple peroral intestinal biopsy capsule gave a fresh impetus to the study of intestinal disorders. During the past five years conflicting reports on intestinal reaction in patients with ankylostomiasis have appeared in the literature. While some investigators (Sheehy et al., 1962, Salem and Truelove, 1964 ; Chaudhuri and Saha, 1964) have recorded definite and significant evidence of intestinal damage in this condition, others have failed to do so (Gilles et al., 1964 ; Layrisse et al., 1964). The present study was therefore undertaken to obtain information about intestinal structure and function in subjects with ankylostomiasis infestation.

\section{Selection of Cases and Methods}

Twenty-two adult males, whose stools were positive for hookworm ova and who had no other associated disease, formed the subjects for this study. Their ages ranged from 18 to 25 years. Nineteen non-infected healthy adult males were studied as controls. Their ages ranged from 20 to 35 years. Both these groups belonged to the lower socio-economic strata of the rural population. In addition to clinical examination each patient had his haemoglobin concentration determined and blood smear examined by standard methods (Dacie, 1956; A.F.I.P. Laboratory, 1924). In all cases bone-marrow studies were carried out and a fat-balance test was done on a diet containing $100 \mathrm{~g}$. of fat. After an initial period of stabilization over five days the faeces were collected for 48 hours for analysis of fat by the method of Van de Kamer et al. (1949). A 5-g. D-xylose-absorption test was done according to the method of Roe and Rice (1948). A jejunal biopsy specimen was taken by Rubin's multipurpose capsule $5-10 \mathrm{~cm}$. distal to the ligament of Treitz. The dietary history of the patients was recorded according to the Indian standards worked out by the Indian Council of Medical Research (I.C.M.R., 1963).

\section{Results}

The clinical observations are recorded in the Table. It was noted that about one-third of the patients had frequent loose stools, though none of them gave a characteristic description suggestive of steatorrhoea. Pitting oedema of the lower extremities was observed in three of the patients.

* Assistant Professor, Department of Medicine, All India Institute of Medical Sciences, New Delhi.

† Postgraduate Student, Department of Medicine, All India Institute of Medical Sciences, New Delhi.

₹ Assistant Professor, Department of Pathology, All India Institate of Medical Sciences, New Delhi.

$\checkmark$ Assistant Professor, Department of Pathology. All India Institute of Medical Sciences, New Delh: 